\title{
THE BOUNDARY PROBLEM ASSOCIATED WITH A DIFFERENTIAL EQUATION IN WHICH THE COEFFICIENT OF THE PARAMETER CHANGES SIGN*
}

\author{
BY \\ RUDOLPH E. LANGER
}

1. Introduction. The boundary value and expansion problem associated with the ordinary linear differential system

$$
\begin{gathered}
u^{\prime \prime}(x)+p(x) u^{\prime}(x)+[q(x)+\lambda k(x)] u(x)=0, \\
L_{j}(u)=0, \quad j=1,2,
\end{gathered}
$$

with coefficients real and continuous, and $\lambda$ a complex parameter, has been extensively studied. The results of these investigations (and of those dealing with the generalized system of order $n$ ) comprise an extensive theory for the case in which the coefficient of the parameter maintains its sign in the interval determined by the boundary conditions. Not merely the existence, but the asymptotic forms of the characteristic values and functions are known, and the expansibility of a function, arbitrary within wide restrictions, in a series of characteristic functions has been established.

Far less has been done with the cases in which $k(x)$ either changes sign or otherwise vanishes in the given interval. The existence of infinitely many characteristic values has been variously established, $\dagger$ but their distribution and the form of the corresponding solutions has not been determined. If $k(x)$ changes sign a finite number of times Hilbert's theory of the polar integral equation is applicable and yields the theorem that a function which is continuous together with its first four derivatives and which satisfies the boundary and certain auxiliary conditions is expansible in a uniformly convergent series of characteristic functions. The method of infinitely many variables has been applied with considerable success to the more general case in which $k(x)$ is less restricted. $\ddagger$ Still the expansion theorems obtained are hardly comparable with those which have been obtained under the hypothesis that the sign of $k(x)$ does not change.

* Presented to the Society, September 8, 1927; received by the editors January 17, 1928.

$\dagger$ For references see the introduction to the paper by L. Lichtenstein, Zur Analysis der unendlichvielen Variabeln, Rendiconti del Circolo Matematico di Palermo, vol. 38 (1914), p. 113.

$\ddagger$ Lichtenstein, loc. cit., also Anna Pell Wheeler, Linear ordinary self-adjoint differential equations of the second order, American Journal of Mathematics, vol. 46 (1927), p. 309. 
The present paper is devoted to a discussion of the case in which the coeffcient $k(x)$ is the function $x^{x}$, where $\nu$ is any real positive constant and the interval includes the point $x=0$. If $\nu$ is integral this is a specialization of the equation discussed above since the coefficient remains real. If the integer is even or $x=0$ is an end point of the interval the coefficient merely vanishes, while if $\nu$ is an odd integer a change of sign takes place. If $\nu$ is not integral the coefficient clearly changes from real to complex.

The method used is that of asymptotic forms. The equation is solved in terms of Bessel's functions and the asymptotic forms of the characteristic values and of the characteristic functions are deduced. It is shown that if $f(x)$ is any integrable function then the expansion in terms of the characteristic functions converges except at the end points to the average $\frac{1}{2}[f(x+)+f(x-)]$ in any interval in which $f(x)$ is of bounded variation and $x \neq 0$. If $f(x)$ is of bounded variation throughout the whole interval the expansion converges also at $x=0$, to the average of the functional values if $\nu$ is an integer and otherwise to a weighted average.

2. The solution of the equation. We consider the equation

$$
u^{\prime \prime}+\lambda x^{\nu} u(x)=0,
$$

in which $\nu$ is any real positive constant and $\lambda$ is a complex parameter. When $x<0$ we specify the symbol $x^{\nu}$ to designate $|x|^{v} e^{\nu x i}$. Similarly $\rho^{1 / 2}$ shall designate $|\rho|^{1 / 2} e^{(i / 2) \text { arg } \rho}$.

It is convenient to set

$$
\nu=2 k-2, \quad \lambda=k^{2} \rho^{2},
$$

and to consider $\rho$ as a new parameter. If the variables are then changed by the substitutions

$$
y(x)=x^{-1 / 2} u(x), \quad t=\rho x^{k},
$$

the equation takes the form of the Bessel's equation with solutions

$$
y_{j}(t)=H_{1 /(2 k)}^{(j)}(t),{ }^{*} \quad j=1,2 .
$$

Hence we may choose as a set of independent solutions of the equation (1)

$$
u_{j}(x, \rho)=\left(\frac{\pi \rho}{2}\right)^{1 / 2} e^{ \pm(k+1) \pi i /(4 k)} x^{1 / 2} H_{1 /(2 k)}^{(j)}\left(\rho x^{k}\right), \quad j=1,2 . \dagger
$$

* The choice of the Bessel's functions of the third kind is made because of their suitability for the subsequent developments. Cf. Watson, Theory of Bessel's Functions, Cambridge University Press, 1922, p. 73. This reference will be indicated in the text by W.

$\dagger$ In combining two formulas by the use of double signs we shall agree to associate the upper signs with $j=1$ and the lower signs with $j=2$. 
With the use of the formula [W, p. 74]

we obtain from (3)

$$
\frac{d}{d t}\left\{t_{r}^{r} H_{r}^{(j)}(t)\right\}=t^{r} H_{r-1}^{(j)}(t)
$$

(3a) $u_{i}^{\prime}(x, \rho)=k\left(\frac{\pi}{2}\right)^{1 / 2} e^{ \pm(k+1) \pi i /(4 k)} \rho^{3 / 2} x^{k-1 / 2} H_{1 /(2 k)-1}^{(j)}\left(\rho x^{k}\right), \quad j=1,2$.

3. The asymptotic forms of the solutions. If we adopt as generic symbols $B(t)$ to designate a bounded function, and $I_{p}(t, r)$ to designate a function of the form

where

$$
I_{p}(t, r)=\sum_{m=0}^{p-1} \frac{(r, m)}{(-2 i t)^{m}}+\frac{B(t)}{t^{p}}
$$

the asymptotic expressions

$$
(r, m)=\frac{\Gamma\left(r+m+\frac{1}{2}\right)}{m ! \Gamma\left(r-m+\frac{1}{2}\right)}
$$

$$
H_{r}^{(j)}(t)=\left(\frac{2}{\pi t}\right)^{1 / 2} e^{ \pm i(t-r \pi / 2-\pi / 4)} I_{p}( \pm t, r)
$$

are available [W, p. 198] and are valid for any integer $p>0$, and uniformly so for $-\pi+\epsilon \leqq \arg t \leqq \pi-\epsilon$, where $\epsilon$ is an arbitrarily small positive constant. We find from this that with the use of the abbreviations

$$
\begin{aligned}
I(t) & \equiv I_{p}\left(t, \frac{1}{2 k}\right), \\
\theta_{j}(x, \rho) & \equiv x^{->/ 4} e^{ \pm i \rho x k} I\left( \pm \rho x^{k}\right), \quad j=1,2,
\end{aligned}
$$

we may write

$$
\begin{gathered}
u_{j}(x, \rho)=\theta_{j}(x, \rho), \\
x>0,-\pi+\epsilon \leqq \arg \rho \leqq \pi-\epsilon .
\end{gathered}
$$

for

Similarly we deduce as the formula for the derivative

$$
u_{j}^{\prime}(x, \rho)= \pm i \rho k x^{p / 4} e^{ \pm i_{\rho} x k} I_{p}\left(t, \frac{1}{2 k}-1\right) .
$$

To obtain the asymptotic forms when $x<0$ we employ the formulas [W, p. 75]

$$
H_{r}^{(j)}\left(t e^{m \pi i}\right)=\frac{\sin (1 \mp m) r \pi}{\sin r \pi} H_{r}^{(j)}(t) \mp e^{\mp r \pi i} \frac{\sin m r \pi}{\sin r \pi} H_{r}^{(8-j)}(t),
$$


which are valid when $m$ is an integer. Setting $|x|=\xi$, i.e. $x=\xi e^{r i}$, and defining the integer $K$ and the fractions $\eta, \eta^{\prime}$ by the relations

$$
\begin{gathered}
K-1<k \leqq K, \\
k=K-\eta=(K-1)+\eta^{\prime},
\end{gathered}
$$

we find from (3) and (6) that if

$$
\rho^{\prime}=\rho e^{-\eta \pi i}
$$

and $\arg \rho^{\prime}$ is restricted to the interval $(-\pi+\epsilon, \pi-\epsilon)$, then

$$
u_{j}(x, \rho)=i^{1+\eta}\left[c_{j 1} \theta_{1}\left(\xi, \rho^{\prime}\right)+c_{j 2} \theta_{2}\left(\xi, \rho^{\prime}\right)\right], \quad j=1,2,
$$

where

$$
\begin{gathered}
c_{11}=-\cos \frac{\eta^{\prime} \pi}{2 k} \csc \frac{\pi}{2 k}, \quad c_{12}=c_{21}=-i \cos \frac{\eta \pi}{2 k} \csc \frac{\pi}{2 k}, \\
c_{22}=\cos \frac{(1+\eta) \pi}{2 k} \csc \frac{\pi}{2 k} .
\end{gathered}
$$

In terms of $x$ formula (9) is

$$
u_{j}(x, \rho)=i^{K} x^{-\nu / 4}\left[c_{j l} e^{i \rho x k} I\left(\rho x^{k}\right)+c_{j, 3-l} e^{-i \rho x k} I\left(-\rho x^{k}\right)\right], \quad j=1,2,
$$

for

$$
x<0, \quad(-1+\eta) \pi+\epsilon \leqq \arg \rho \leqq(1+\eta) \pi-\epsilon,
$$

where $l=1$ if $K$ is even, and $l=2$ if $K$ is odd.

In (11) as in (5), (5a) the asymptotic form of $u_{j}^{\prime}$ may be obtained from that of $u_{i}$ by differentiating only the exponential factors and replacing $I(t)$ by $I_{p}(t, 1 /(2 k)-1)$.

When $\nu$ is an integer it follows from the work of Birkhoff* that for $\rho$ confined to any quadrant formed by the axes of reals and imaginaries every solution of equation (1) is asymptotically of the form

$$
u(x)=x^{-\nu / 4}\left[a_{1} e^{i \rho x k} v_{1}(x, \rho)+a_{2} e^{-i \rho x k} v_{2}(x, \rho)\right],
$$

for $x \geqq \delta>0$, while for $x \leqq-\delta$ such a solution is of similar structure with $a_{1}$ and $a_{2}$ replaced by different constants $\bar{a}_{1}$ and $\bar{a}_{2}$ respectively. From formulas (5) and (11) the relation between these sets of constants for the quadrants in which (5) and (11) are both valid is found to be

$$
\begin{aligned}
& \bar{a}_{1}=i^{K}\left(c_{1, l} a_{1}+c_{2, l} a_{2}\right), \\
& \bar{a}_{2}=i^{K}\left(c_{1,3-l} a_{1}+c_{2,3-l} a_{2}\right) .
\end{aligned}
$$
219-231.

* Birkhoff, Asymptotic solutions of differential equations, these Transactions, vol. 9 (1908), pp. 
The asymptotic expressions obtained display the functional form of the solutions when $\left|\rho x^{k}\right|$ is sufficiently large. For small values of $\left|\rho x^{k}\right|$ we observe that since

$$
t^{\mu} H_{ \pm \mu}^{(i)}(t), \quad j=1,2, \quad \mu>0,
$$

is bounded when $t$ is bounded, it follows from (3) and (3a) that

$$
\begin{aligned}
\left|x^{\prime / 4} u_{j}(x, \rho)\right| & <M_{1}, \\
\left|u_{j}^{\prime}(x, \rho)\right| & <\dot{M}_{2}|\rho|^{1 / 2+1 /(2 k)}, \quad \text { for }\left|\rho x^{k}\right|<M .
\end{aligned}
$$

Combining these inequalities with formulas (5) and (5a) we find that

$$
\begin{aligned}
u_{j}(x, \rho) & =x^{-\nu / 4} e^{ \pm i \rho x k} B(x, \rho), \\
u_{j}^{\prime}(x, \rho) & = \pm i k \rho e^{ \pm i \rho x k} B(x, \rho),
\end{aligned}
$$

Lastly we obtain from the formulas [W, pp. 74, 40]

$$
\lim _{t \rightarrow 0} t^{\mu} H_{\mu}^{(j)}(t)=A_{j}, \quad \lim _{t \rightarrow 0} t^{\mu} H_{-\mu}^{(j)}(t)=A_{j} e^{ \pm \mu \pi i}, \quad \mu>0,
$$

where

and

$$
A_{i}=\frac{ \pm 2^{\mu}}{i \Gamma(1-\mu) \sin \mu \pi}
$$

$$
\Gamma(z) \Gamma(1-z)=\frac{\pi}{\sin \pi z},
$$

and the relations (3) and (3a)

$$
\begin{aligned}
u_{j}(0, \rho) & =\frac{\mp i}{\pi^{1 / 2}} e^{ \pm(k+1) \pi i /(4 k)} \Gamma\left(\frac{1}{2 k}\right)\left(\frac{\rho}{2}\right)^{1 / 2-1 /(2 k)}, \\
u_{j}^{\prime}(0, \rho) & =\frac{ \pm 2 k i}{\pi^{1 / 2}} e^{ \pm(k-1) \pi i /(4 k)} \Gamma\left(1-\frac{1}{2 k}\right)\left(\frac{\rho}{2}\right)^{1 / 2+1 /(2 k)}, j=1,2 .
\end{aligned}
$$

4. The boundary problem. The characteristic values. We consider now the boundary problem obtained by adjoining to equation (1) the boundary conditions

$$
u(\beta)=0, \quad u(-\alpha)=0,{ }^{*} \quad \alpha>0, \quad \beta>0 .
$$

Since every solution of (1) may be written in the form

$$
u(x)=a_{1} u_{1}(x)+a_{2} u_{2}(x)
$$

* For brevity we shall use the notation $u(x)$ in place of $u(x, \rho)$ when no confusion is apt to result. 
it is clear that there exists a solution satisfying conditions (16) if and only if the value of $\rho$ is such that

$$
\Omega(\beta,-\alpha)=0,
$$

where

$$
\Omega(s, t)=\left|\begin{array}{ll}
u_{1}(s) & u_{2}(s) \\
u_{1}(t) & u_{2}(t)
\end{array}\right| .
$$

Since by (2) any half $\rho$-plane maps into the entire $\lambda$-plane we may restrict ourselves, in seeking the roots of equation (18), to any half-plane

$$
-\delta \leqq \arg \rho \leqq \pi-\delta, \text { where } 0<\delta<\eta^{\prime} \pi .
$$

For such values the asymptotic forms of $u_{j}(x, \rho)$ and of $u_{i}\left(x, \rho^{\prime}\right)$ are given by formulas (5) when $x>0$. We must distinguish between the cases in which $\nu$ is or is not an even integer.

Case 1. $\nu$ not an even integer. In this case the rays

$$
\begin{aligned}
\arg \rho & =0, \\
\arg \rho^{\prime} & =0, \text { i.e. } \quad \arg \rho=\eta \pi,
\end{aligned}
$$

are distinct. Suppose now that $\rho$ is confined to a sector $S_{1}$ of the half-plane (19) which includes the ray (20) but excludes the ray (21). In such a sector $R\left(i \rho^{\prime}\right)>0$, and from (4) $\theta_{2}\left(x, \rho^{\prime}\right)$ is asympţotically negligible in comparison with $\theta_{1}\left(x, \rho^{\prime}\right)$ for $x>0$. Hence we find in this sector

$$
\Omega(\beta,-\alpha) \sim i^{1+\eta}\left|\begin{array}{ll}
\theta_{1}(\beta, \rho) & \theta_{2}(\beta, \rho) \\
c_{11} \theta_{1}\left(\alpha, \rho^{\prime}\right) & c_{21} \theta_{1}\left(\alpha, \rho^{\prime}\right)
\end{array}\right|^{*}
$$

and the equation (18) takes the form

$$
c_{21} e^{i \rho \beta k} I\left(\rho \beta^{k}\right)-c_{11} e^{-i \rho \beta} I\left(-\rho \beta^{k}\right)=0 .
$$

If we set

$$
r_{1, m}=\beta^{-k}\left[\left(m-\frac{1}{4}\right) \pi+\frac{1}{2 i} \log \left(\cos \frac{\eta^{\prime} \pi}{2 k} \sec \frac{\eta \pi}{2 k}\right)\right],
$$

and observe that

$$
\log I\left(-\rho \beta^{k}\right)-\log I\left(\rho \beta^{k}\right)=\frac{\left(\frac{1}{2 k}, 1\right)}{i_{\rho} \beta^{k}}+\frac{B(\rho)}{\rho^{3}},
$$

* We use the symbol $\sim$ to indicate that the functions are of the same asymptotic form. Thus if in this formula the sign $\sim$ were to be replaced by $=$ it would be necessary to replace in the right hand member the quantity $i^{1+\eta} \theta_{2}\left(\alpha \rho^{\prime}\right)\left[c_{22} \theta_{1}(\beta)+c_{12} \theta_{2}(\beta)\right]$, which has been dropped. By (4) it is evident, however, that this quantity is of lower order of magnitude when $|\rho|$ is large than the error due to stopping at any definite term of the asymptotic expansion of the expression retained. 
we may write (22) in the form

$$
\rho-\left[r_{1, m}+\frac{\left(\frac{1}{2 k}, 1\right)}{2 \rho \beta^{2 k}}+\frac{B(\rho)}{\rho^{3}}\right]=0 .
$$

Let the points $r_{1, m}$ in the $\rho$-plane be surrounded now by small circles with centers at $r_{1, m}$ and radius $\epsilon$. Then for $m$ sufficiently large the parenthesis in (22a) will have a value lying within this small circle for every value of $\rho$ on the circumference, and as $\rho$ traces the circumference the argument of the left hand member of (22a) increases by $2 \pi$. Hence (22a) has just one root $\rho_{1, m}$ within the small circle. From (22a) we may write then

$$
\rho_{1, m}=r_{1, m}+B(m) / m \text {. }
$$

Computing the term in $1 / \rho$ from this and observing that $r_{1, m}$ is of the order of $m$ we obtain the formula

$$
\rho_{1, m}=r_{1, m}+\frac{\left(\frac{1}{2 k}, 1\right)}{2 r_{1, m} \beta^{2 k}}+\frac{B(m)}{m^{3}} .
$$

In similar manner if $\rho$ is restricted to a sector $S_{2}$ of the half-plane (19) which includes the ray (21) but excludes the ray (20) we have $R(i \rho)<0$ and

$$
\Omega(\beta,-\alpha) \sim-\theta_{2}(\beta, \rho) \theta_{1}(-\alpha, \rho) .
$$

The characteristic equation (18) now takes the form

$$
c_{11} e^{i \rho^{\prime} \alpha^{k}} I\left(\rho \alpha^{k}\right)+c_{12} e^{-i \rho^{\prime} \alpha^{k}} I\left(-\rho^{\prime} \alpha^{k}\right)=0 .
$$

If we set

$$
r_{2, m}=\alpha^{-k}\left[\left(m-\frac{1}{4}\right) \pi-\frac{1}{2 i} \log \left(\cos \frac{\eta^{\prime} \pi}{2 k} \sec \frac{\eta \pi}{2 k}\right)\right],
$$

we find precisely as in the preceding discussion, if we recall (8), that

$$
\rho_{2, m}=e^{\eta \pi i}\left[r_{2, m}+\frac{\left(\frac{1}{2 k}, 1\right)}{2 r_{2, m} \alpha^{2 k}}+\frac{B(m)}{m^{3}}\right] .
$$

We may state then the following theorem:

THEOREM 1. If in equation (1) $\nu$ is not an even integer the infinitely many characteristic values of $\rho$ are asymptotically spaced at regular intervals along two distinct straight lines in the complex $\rho$-plane, the formulas for these values being given by (24) and (27) above. 
We observe in particular that when $\nu$ is an odd integer then $\eta=\eta^{\prime}=\frac{1}{2}$, and the logarithmic terms in (23) and (26) vanish, so that $\rho_{1, m}$ is asymptotically real and $\rho_{2, m}$ asymptotically pure imaginary.* In this case we find from (2) the theorem

THEOREM 1a. If in equation (1) $\nu$ is an odd integer the infinitely many characteristic values are given asymptotically by the formulas

$$
\begin{aligned}
& \lambda_{1, m}=k^{2} \beta^{-2 k}\left[\left(m-\frac{1}{4}\right)^{2} \pi^{2}+\left(\frac{1}{2 k}, 1\right)\right]+\frac{B(m)}{m^{2}}, \\
& \lambda_{2, m}=-k^{2} \alpha^{-2 k}\left[\left(m-\frac{1}{4}\right)^{2} \pi^{2}+\left(\frac{1}{2 k}, 1\right)\right]+\frac{B(m)}{m^{2}} .
\end{aligned}
$$

Case 2. $\nu$ an even integer. In this case we find from (2), (7), and (9) that $k$ is an integer and hence that $K=k, \eta=0, \eta^{\prime}=1$, and $\rho^{\prime}=\rho$. From the form

$$
\begin{aligned}
& \Omega(\beta,-\alpha)=i\left[c_{21} \theta_{1}(\beta) \theta_{1}(\alpha)+c_{22} \theta_{1}(\beta) \theta_{2}(\alpha)\right. \\
& \left.-c_{11} \theta_{2}(\beta) \theta_{1}(\alpha)-c_{12} \theta_{2}(\beta) \theta_{2}(\alpha)\right]
\end{aligned}
$$

the equation (18) becomes

$$
\begin{aligned}
& e^{i \rho(\beta k+\alpha k)} I\left(\rho \beta^{k}\right) I\left(\rho \alpha^{k}\right)+i\left(\cos \frac{\pi}{2 k}\right) e^{i \rho(\beta k-\alpha k)} I\left(\rho \beta^{k}\right) I\left(-\rho \alpha^{k}\right) \\
& +i\left(\cos \frac{\pi}{2 k}\right) e^{-i \rho(\beta k-\alpha k)} I\left(-\rho \beta^{k}\right) I\left(\rho \alpha^{k}\right)-e^{-i \rho(\beta k+\alpha k)} I\left(-\rho \beta^{k}\right) I\left(-\rho \alpha^{k}\right)=0 .
\end{aligned}
$$

The equation of this type has been studied, $\dagger$ and it is known (i) that its roots are asymptotic to those of the equation

$$
e^{2 i \rho(\beta k+\alpha k)}+i\left(\cos \frac{\pi}{2 k}\right) e^{2 i \rho \beta k}+i\left(\cos \frac{\pi}{2 k}\right) e^{2 i \rho \alpha k}-1=0,
$$

and (ii) that the roots of this latter equation lie in a strip bounded by parallels to the axis of reals, $\ddagger$ and that for $|\rho|>N$ at most three roots lie between any two lines perpendicular to this axis and less than the distance

apart.

$$
\frac{2 \pi \alpha^{k}}{\beta^{k}+\alpha^{k}}
$$

* It is known, of course, that $\rho_{1, m}$ and $\rho_{2, m}$ are actually real and pure imaginary respectively. Cf. Ince, Ordinary Differential Equations, London, 1927, p. 238.

$\dagger$ Tamarkin, Some General Problems of the Theory of Linear Differential Equations etc. (in Russian), Petrograd, 1917, also C. E. Wilder, these Transactions, vol. 18 (1917), p. 420.

‡ They are actually real. See note ${ }^{*}$ above. 
We observe that in the particular cases in which $\beta^{k}$ and $\alpha^{k}$ are commensurable, say

$$
\beta^{k}=(p / q) \alpha^{k},
$$

where $p$ and $q$ are integers, the solution of equation (29a) is an algebraic problem, the equation being algebraic of the degree $p+q$ in $e^{2 i \rho \alpha^{k} q^{-1}}$. Denoting the roots of this equation by $r_{j}, j=1,2, \cdots, p+q$, we have then

$$
e^{2 i \rho \alpha k / q}=r_{j},
$$

from which the characteristic values are found to be

where

$$
\rho_{j, m}=q \alpha^{-k}\left[m \pi+\frac{1}{2 i} \log r_{j}+\epsilon_{j}(m)\right]
$$

$$
\lim _{m \rightarrow \infty} \epsilon_{j}(m)=0 .
$$

We consider more explicitly the particular case in which

$$
\alpha=\beta \text {. }
$$

The determination of the characteristic values is in this case easily possible for the equation (28) reduces to the quadratic

$$
\theta_{1}^{2}(\beta)+2 i\left(\cos \frac{\pi}{2 k}\right) \theta_{1}(\beta) \theta_{2}(\beta)-\theta_{2}^{2}(\beta)=0,
$$

which we write in the form

$$
\left\{\theta_{1}(\beta)+i e^{\pi i /(2 k)} \theta_{2}(\beta)\right\}\left\{\theta_{1}(\beta)+i e^{-\pi i /(2 k)} \theta_{2}(\beta)\right\}=0 .
$$

From this the characteristic values are found directly to be

$$
\rho_{j, m}=\beta^{-k}\left[\left(m-\frac{1}{4} \pm \frac{1}{4 k}\right) \pi-\frac{\left(\frac{1}{2 k}, 1\right)}{2\left(m-\frac{1}{4} \pm \frac{1}{4 k}\right) \pi}\right]+\frac{B(m)}{m^{2}}, j=1,2,
$$

and we have the following theorem:

ThEOREM 2. If in equation (1) $\nu$ is an even integer and $\alpha=\beta$, the infinitely many characteristic values are given asymptotically by the formula

$$
\lambda_{j, m}=k^{2} \beta^{-2 k}\left[\left(m-\frac{1}{4} \pm \frac{1}{4 k}\right)^{2} \pi^{2}-\left(\frac{1}{2 k}, 1\right)\right]+\frac{B(m)}{m^{2}}, \quad j=1,2 .
$$

It appears from this that the case $\nu$ an even integer is irregular in the 
sense that the characteristic values (32) are not the limiting values which would be obtained from (24) and (27) by allowing $\eta$ to approach zero,i.e. by allowing $\nu$ to approach the even integer.

5. The characteristic functions. When $\rho$ is a characteristic value the constants $a_{1}, a_{2}$ in (17) may be chosen to make the solution satisfy the boundary conditions. The solution (17) becomes then a characteristic function. We proceed to derive its asymptotic form. For convenience we shall omit the subscripts on $\rho$, understanding that in the discussion of this section $\rho$ is always taken to be a characteristic value.

Case 1. $\nu$ not an even integer. Consider first the function corresponding to $\rho=\rho_{1, m}$. Choosing the constants in (17) thus:

we find

$$
a_{1}=-u_{2}(\beta, \rho), \quad a_{2}=u_{1}(\beta, \rho),
$$

$$
u(x)=\Omega(\beta, x, \rho) .
$$

The characteristic equation (22) is in this case

$$
c_{11} \theta_{2}(\beta, \rho)-c_{21} \theta_{1}(\beta, \rho) \sim 0,
$$

and substituting from this in (34) we find

$$
u(x) \sim-\left(\frac{c_{21}}{c_{11}}\right)^{1 / 2} \theta_{1}(\beta, \rho)\left[\left(\frac{c_{21}}{c_{11}}\right)^{1 / 2} \theta_{1}(x, \rho)-\left(\frac{c_{11}}{c_{21}}\right)^{1 / 2} \theta_{2}(x, \rho)\right] .
$$

With the use of the formulas (4) the form of $u(x)$ when $x>0$ is thus obtained.

If $x<0$ we observe that because of (18) we may write

$$
\Omega(\beta, x, \rho)=\frac{u_{1}(\beta, \rho)}{u_{1}(-\alpha, \rho)} \Omega(-\alpha, x, \rho) .
$$

Substituting from (9) for $u_{j}(x)$ and $u_{j}(-\alpha)$, and observing that

$$
c_{11} c_{22}-c_{12} c_{21}=1 \text {, }
$$

we derive the result

$$
u(x, \rho)=\frac{i^{1+\eta} \theta_{1}(\beta, \rho) .}{c_{11} \theta_{1}\left(\alpha, \rho^{\prime}\right)+c_{12} \theta_{2}\left(\alpha, \rho^{\prime}\right)} \Omega\left(\alpha, \xi, \rho^{\prime}\right) .
$$

Now when $\rho=\rho_{1, m}, \theta_{2}\left(\alpha, \rho^{\prime}\right)$ is asymptotically negligible in comparison with $\theta_{1}\left(\alpha, \rho^{\prime}\right)$ and hence we may replace the denominator of (37) by $c_{11} \theta_{1}\left(\alpha, \rho^{\prime}\right)$. Lastly if we remove from (35) and (37) the factor

$$
-2 i\left(\frac{c_{21}}{c_{11}}\right)^{1 / 2} \theta_{1}(\beta, \rho),
$$

which is independent of $x$, we have the following theorem: 
THEOREM 3. If in equation (1) $\nu$ is not an even integer the characteristic function $u_{1, m}(x)$ corresponding to the characteristic value $\rho_{1, m}$ is of the asymptotic form

$$
u_{1, m}(x) \sim \frac{x^{-x / 4}}{2 i}\left[\left(\frac{c_{21}}{c_{11}}\right)^{1 / 2} e^{i \rho x k} I\left(\rho x^{k}\right)-\left(\frac{c_{11}}{c_{21}}\right)^{1 / 2} e^{-i \rho x k} I\left(-\rho x^{k}\right)\right]
$$

when $x>0$,

$$
u_{1, m}(x) \sim \frac{\xi^{-\nu / 4}}{-2 i^{-\eta}\left(c_{11} c_{21}\right)^{1 / 2}}\left[e^{-i \rho^{\prime} \xi k} I\left(-\rho^{\prime} \xi^{k}\right)-e^{-i \rho^{\prime}\left(2 \alpha^{k-\xi k)}\right.} \frac{I\left(-\rho^{\prime} \alpha^{k}\right)}{I\left(\rho^{\prime} \alpha^{k}\right)} I\left(\rho^{\prime} \xi^{k}\right)\right]
$$

when $x<0, \rho=\rho_{1, m}$.

To derive the form of $u_{2, m}(x)$ we consider the solution

$$
u(x)=-i^{-2 \eta} \Omega(x,-\alpha, \rho) .
$$

When $x<0$ we obtain from this by means of the equations (9) and (36)

$$
u(x)=\Omega\left(\xi, \alpha, \rho^{\prime}\right) .
$$

The characteristic equation in this case is (25), i.e.

$$
c_{11} \theta_{1}\left(\alpha, \rho^{\prime}\right)+c_{12} \theta_{2}\left(\alpha, \rho^{\prime}\right) \sim 0 .
$$

Observing that (38) and (25) may be obtained from (34) and (22) by interchanging the following letters:

(39) $u_{1}$ with $u_{2}, \theta_{1}$ with $\theta_{2}, c_{21}$ with $-c_{12}, \beta$ with $\alpha, \rho$ with $\rho^{\prime}, x$ with $\xi$, it is clear that the result, namely the form of $u(x)$ when $x<0$, may be similarly obtained from (35).

When $x>0$ we have because of (18) the relations

$$
\Omega(x,-\alpha, \rho)=\frac{u_{2}(-\alpha, \rho)}{u_{2}(\beta, \rho)} \Omega(x, \beta, \rho)=\frac{i^{1+\eta} \theta_{2}\left(\alpha, \rho^{\prime}\right)}{c_{11} \theta_{2}(\beta, \rho)-c_{21} \theta_{1}(\beta, \rho)} \Omega(x, \beta, \rho),
$$

and hence from (38)

$$
u(x)=-\frac{i^{1-\eta \theta_{2}}\left(\alpha, \rho^{\prime}\right)}{c_{11} \theta_{2}(\beta, \rho)-c_{21} \theta_{1}(\beta, \rho)} \Omega(x, \beta, \rho) .
$$

Observing that the interchanges of letters (39) lead from (37) to (40) except for a factor $-i^{-2 n}$ it is clear that the result is again derivable from that of the preceding discussion. Removing the factor

$$
2 i\left(\frac{-c_{12}}{c_{11}}\right)^{1 / 2} \theta_{2}\left(\alpha, \rho^{\prime}\right)
$$


from the solution considered we may state the following theorem:

THEOREM 3. If in equation (1) $\nu$ is not an even integer the asymptotic forms of the characteristic functions $u_{2, m}(x)$ corresponding to $\rho=\rho_{2, m}$ are given by the formulas

$$
u_{2, m}(x) \sim \frac{x^{-\nu / 4}}{-2 i^{\eta}\left(-c_{11} c_{12}\right)^{1 / 2}}\left[e^{i \rho x k} I\left(\rho x^{k}\right)-e^{i \rho(2 \beta k-x k)} \frac{I\left(\rho \beta^{k}\right)}{I\left(-\rho \beta^{k}\right)} I\left(-\rho x^{k}\right)\right]
$$

when $x>0$,

$$
u_{2, m}(x) \sim \frac{\xi^{-p / 4}}{2 i}\left[\left(\frac{c_{11}}{-c_{12}}\right)^{1 / 2} e^{i \rho^{\prime} \xi k} I\left(\rho^{\prime} \xi^{k}\right)-\left(\frac{-c_{12}}{c_{11}}\right)^{1 / 2} e^{-i \rho^{\prime} \xi k} I\left(-\rho^{\prime} \xi^{k}\right)\right]
$$

when $x<0, \rho=\rho_{2, m}$.

In particular if $\nu$ is an odd integer these forms may be somewhat reduced and we have the following theorem:

THEOREM 3a. If in equation (1) $\nu$ is an odd integer the characteristic functions $u_{1, m}(x)$ corresponding to the characteristic values $\rho=\rho_{1, m}$ are given by the formulas

when $x>0$,

$$
\begin{array}{r}
u_{1, m}(x) \sim x^{-v / 4}\left[\left\{1+\frac{B\left(m x^{k}\right)}{\left(m x^{k}\right)^{2}}\right\} \sin \left(\rho_{1, m} x^{k}+\frac{\pi}{4}\right)\right. \\
\left.+\frac{B\left(m, x^{k}\right)}{m x^{k}} \cos \left(\rho_{1, m} x^{k}+\frac{\pi}{4}\right)\right],
\end{array}
$$

when $x<0$.

$$
\begin{aligned}
u_{1, m}(x) \sim-\xi^{-\nu / 4}\left(\sin \frac{\pi}{4 k}\right) & {\left[e^{-\rho_{1, m} \xi k}\left\{1+\frac{B\left(m \xi^{k}\right)}{m \xi^{k}}\right\}\right.} \\
& \left.-e^{-\rho_{1, m}(2 \alpha k-\xi k)}\left\{1+\frac{B\left(m \xi^{k}\right)}{m \xi^{k}}\right\}\right],
\end{aligned}
$$

The form of the characteristic function $u_{2, m}(x)$ for $x>0$ (or $x<0$ ) is obtainable from that of $u_{1, m}(x)$ for $x<0$ (or $\left.x>0\right)$ by interchanging $x$ with $\xi$ and $\alpha$ with $\beta$.

From this we see that for large values of $m$ any solution has the character of $|x|^{-\nu / 4} \sin \left(\rho_{1, m}|x|^{k}+\pi / 4\right)$ in any interval of the $x$-axis lying entirely on one side of the origin, while in any interval of the $x$-axis on the other side of the origin the graphs of the solutions lie arbitrarily close to the axis for $m$ sufficiently large.

It is desirable also to note the values taken on by the characteristic functions at $x=0$. Removing the constant factors specified in the discussion above and substituting from (15) we find 


$$
u_{j, m}(0)=C_{j} \rho_{i, m}^{1 / 2-1 /(2 k)}, \quad u_{i, m}^{\prime}(0)=C_{j}^{\prime} \rho_{j, m}^{1 / 2+1 /(2 k)}, \quad j=1,2,
$$

where $C_{i}$ and $C_{i}^{\prime}$ are constants independent of $m$. Since $\left|\rho_{j, m}\right|$ is of the order $m$ it is seen that $\left|u_{j, m}(0)\right|$, and $\left|u_{i, m}^{\prime}(0)\right|$, become infinite with $m$.

Case 2. $\nu$ an even integer. Inasmuch as we have derived the asymptotic forms of the characteristic values when $\nu$ is even only for the case $\alpha=\beta$, we shall confine our discussion of the characteristic functions also to this case Setting

$$
u(x)=\Omega(\beta, x),
$$

and observing that from (31) we have

$$
\theta_{1}\left(\beta, \rho_{j, m}\right)=-i e^{ \pm \pi i /(2 k)} \theta_{2}\left(\beta, \rho_{j, m}\right), \quad j=1,2,
$$

we obtain directly on dropping the factor

the form

$$
-2 e^{\pi i / 4 \pm \pi i /(4 k)} \theta_{2}\left(\beta, \rho_{i, m}\right)
$$

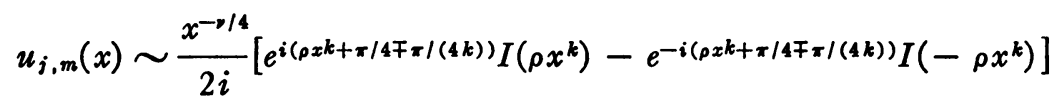

for $x>0, \rho=\rho_{1, m}$, or in an alternative form

$$
\begin{array}{r}
u_{i, m}(x) \sim x^{j / 4}\left[\left\{1+\frac{B\left(m x^{k}\right)}{\left(m x^{k}\right)^{2}}\right\} \sin \left(\rho_{j, m} x^{k}+\frac{\pi}{4} \mp \frac{\pi}{4 k}\right)\right. \\
\left.-\frac{B\left(m x^{k}\right)}{m x^{k}} \cos \left(\rho_{j, m} x^{k}+\frac{\pi}{4} \mp \frac{\pi}{4 k}\right)\right]
\end{array}
$$

for $x>0, j=1,2$.

For $x<0$ we have since $\rho^{\prime}=\rho, \eta=0$,

$$
u(x)=\frac{i \theta_{2}(\beta)}{c_{21} \theta_{1}(\beta)+c_{22} \theta_{2}(\beta)} \Omega(\beta, \xi) .
$$

Substituting in this the values of $c_{21}, c_{22}$ as given by (10) we find that the fraction on the right reduces to $(-1)^{i}$ for $\rho=\rho_{i, m}$. Hence we have on comparing with (42)

$$
u_{i, m}(x)=\mp u_{j, m}(\xi) \text { for } x<0, \quad j=1,2 .
$$

THEOREM 4. If in equation (1) $\nu$ is an even integer and $\alpha=\beta$, the asymptotic forms of the characteristic functions are given for $x>0$ by (44) above. The functions $u_{1, m}(x)$ are odd functions and the functions $u_{2, m}(x)$ are even. 
Proceeding as in the previous case when $x=0$, we find in this case the values

$$
\begin{array}{ll}
u_{1, m}(0)=0, & u_{1, m}^{\prime}(0)=C_{1}^{\prime} \rho_{1, m}^{1 / 2+1 /(2 k)}, \\
u_{2, m}(0)=C_{2} \rho_{2, m}^{1 / 2-1 /(2 k)}, & u_{2, m}^{\prime}(0)=0 .
\end{array}
$$

6. The formal expansion of a function $f(x)$. We shall suppose now, that the characteristic values are arranged and designated by a single subscript so that $\left|\rho_{m-1}\right| \leqq\left|\rho_{m}\right|$. Then two characteristic functions corresponding to distinct characteristic values are found in the usual way to satisfy the relation

$$
\int_{-\alpha}^{\beta} x^{v} u_{n}(x) u_{l}(x) d x=0,
$$$$
l \neq n .
$$

It is clear that $\lambda=0$ is not a characteristic value, since by equation (1) this would imply $u^{\prime \prime}(x) \equiv 0$, and by $(16) u(x) \equiv 0$. Hence we have for any characteristic function $u$ and the corresponding $\lambda$ the relation

$$
x^{\nu} u^{2} \equiv \frac{1}{2 k \lambda} \frac{d}{d x}\left\{\lambda x^{\nu+1} u^{2}+x u^{\prime 2}-u u^{\prime}\right\}
$$

which is an identity since $u(x)$ satisfies (1). Integrating it and applying (16) we obtain

$$
\int_{-\alpha}^{\beta} x^{\prime} u_{n}^{2}(x) d x=\frac{1}{2 k \lambda_{n}}\left[\beta u_{n}^{\prime 2}(\beta)+\alpha u_{n}^{\prime 2}(-\alpha)\right] .
$$

It is readily found by substituting on the right the asymptotic forms of the characteristic functions that this member and hence the integral on the left is different from zero for $n>N$, i.e. for $n>N$ the characteristic values are all simple. If this is true for all values of $n$, which is clearly the case when the characteristic functions are real, i.e. when $\nu$ is an integer, we may derive in the usual way the expansion associated with an arbitrary function $f(x)$, i.e.

where

$$
\lim _{m \rightarrow \infty} \int_{-\alpha}^{\beta} t^{v} f(t) \phi_{m}(x, t) d t
$$

$$
\phi_{m}(x, t)=\sum_{n=1}^{m} \frac{u_{n}(x) u_{n}(t)}{\int_{-\alpha}^{\beta} t^{v} u_{n}^{2}(t) d t} .
$$

Moreover if $G(x, t, \rho)$ is the Green's function of the system (1), (16), and $\gamma$ is any semi-circle in the $\rho$-plane with center at $\rho=0$ which with the diameter 
joining its end points encloses the characteristic values $\lambda_{n}, n=1,2, \cdots, m$, and no others, then by familiar reasoning*

$$
\phi_{m}(x, t)=\frac{k^{2}}{\pi i} \int_{\gamma} \rho G(x, t, \rho) d \rho .
$$

7. The function $G(x, t, \rho)$. To avoid repetition in the discussion of the expansion problem we shall suppose that $x$, which we consider fixed, lies on the interval $0 \leqq x \leqq \beta$. Clearly the deductions and results for an $x<0$ will be entirely similar to those for $x>0$.

If $W\left[u_{2}, u_{1}\right]$ denotes the Wronskian of the functions $u_{2}$ and $u_{1}$, i.e.

$$
W\left[u_{2}, u_{1}\right]=u_{2} u_{1}^{\prime}-u_{1} u_{2}^{\prime},
$$

we find from formulas (3) and (3a)

$$
W\left[u_{2}, u_{1}\right]=\frac{k \pi \rho^{2}}{2} x^{k} W\left[H_{1 /(2 k)}^{(2)}\left(\rho x^{k}\right), H_{1 /(2 k)}^{(1)}\left(\rho x^{k}\right)\right],
$$

and it follows from the known formula for the Wronskian on the right $[\mathrm{W}, \mathrm{p} .76]$ that

$$
W\left[u_{2}, u_{1}\right] \equiv 2 k \rho i \text {. }
$$

The classical formulas for $G(x, t, \rho) \dagger$ reduce therefore for the case in hand to

$$
G(x, t, \rho)=\left\{\begin{array}{l}
\frac{i \Omega(\beta, x) \Omega(t,-\alpha)}{2 k \rho \Omega(\beta,-\alpha)} \text { for } t<x, \\
\frac{i \Omega(\beta, t) \Omega(x,-\alpha)}{2 k \rho \Omega(\beta,-\alpha)} \text { for } t>x .
\end{array}\right.
$$

We observe now that because of the distribution of the characteristic values as shown in $\$ 4$ it is possible to choose in the $\rho$-plane a sequence of circles $\Gamma_{m}:|\rho|=r_{m}$ having the following properties:

(i) as $m \rightarrow \infty$ over positive integral values, $r_{m} \rightarrow \infty$;

(ii) if $\gamma_{m}$ denotes the semi-circle of $\Gamma_{m}$ for which $-\delta \leqq \theta \leqq \pi-\delta$, then $\gamma_{m}$ with the diameter joining its end points includes just $m$ characteristic values;

(iii) there exists a constant $\chi>0$ such that every point of any arc $\boldsymbol{\gamma}_{m}$ is at a distance greater than $\chi$ from any characteristic value.

For the subsequent discussion we must derive the asymptotic forms of

\footnotetext{
* Birkhoff, these Transactions, vol. 9 (1908) p. 379. If some characteristic values are not simple we may consider $\phi_{m}(x, t)$ defined by (48) rather than by (47).

$\dagger$ Birkhoff, loc. cit., p. 378.
} 
$G(x, t, \rho)$ on the semi-circles $\gamma_{m}$. With this in mind we proceed as follows. If $\nu$ is not an even integer let $\gamma_{m}$ be divided into the arcs

$$
\begin{aligned}
& \gamma_{m 1}:-\delta \leqq \theta \leqq 0, \\
& \gamma_{m 2}: \quad 0 \leqq \theta \leqq \eta \pi, \\
& \gamma_{m 3}: \quad \eta \pi \leqq \theta \leqq \pi-\delta .
\end{aligned}
$$

Consider first any portion of $\gamma_{m 2}$, say $\gamma_{m 2}^{\prime}: 0 \leqq \theta \leqq \theta^{\prime}<\eta \pi$. On this arc $R\left(i \rho^{\prime}\right)>0$ and hence we have

$$
\Omega(\beta,-\alpha) \sim-i^{1+{ }^{n} c_{11} \theta_{2}(\beta, \rho) \theta_{1}\left(\alpha, \rho^{\prime}\right)}\left[1-\frac{c_{21} \theta_{1}(\beta, \rho)}{c_{11} \theta_{2}(\beta, \rho)}\right] .
$$

Substituting from (4) in the bracket on the right we obtain for it the expression

$$
\left[1-\frac{c_{21}}{c_{11}} e^{2 \rho_{\rho \beta} k}\left(1+\frac{B(\rho)}{\rho}\right)\right],
$$

and this is clearly asymptotic to 1 except in the neighborhood of $\theta=0$. But it is known* that if $\left|\rho-\rho_{m}\right|>\chi$, where $\rho_{m}$ is any root of the equation obtained by equating the expression (50) to zero, then the expression (50) itself is uniformly bounded from zero. Similarly if we consider $\gamma_{m 2}^{\prime \prime}: 0<\theta^{\prime}$ $\leqq \theta \leqq \eta \pi$, then on this arc $R(i \rho)<0$ and we have

$$
\Omega(\beta,-\alpha) \sim i^{1+\eta} c_{11} \theta_{1}\left(\alpha, \rho^{\prime}\right) \theta_{2}(\beta, \rho)\left[1+\frac{c_{12} \theta_{2}\left(\alpha, \rho^{\prime}\right)}{c_{11} \theta_{1}\left(\alpha, \rho^{\prime}\right)}\right] .
$$

The bracket in this expression is clearly asymptotic to 1 except in the neighborhood of $\theta=\eta \pi$ where it is uniformly bounded from zero since $\left|\rho-\rho_{m}\right|>\chi$. In the same way we find that $\Omega(\beta,-\alpha)$ is uniformly bounded from zero on the $\operatorname{arcs} \gamma_{m 1}$ and $\gamma_{m 3}$, while

$$
\Omega(\beta,-\alpha) \sim\left\{\begin{array}{c}
i^{i+\eta} c_{21} \theta_{1}(\beta, \rho) \theta_{1}\left(\alpha, \rho^{\prime}\right) \text { for }-\delta \leqq \theta \leqq \theta^{\prime \prime}<0, \\
-i^{1+\eta} c_{12} \theta_{2}(\beta, \rho) \theta_{2}\left(\alpha, \rho^{\prime}\right) \text { for } \eta \pi<\theta^{\prime} \leqq \theta \leqq \pi-\delta .
\end{array}\right.
$$

If $\nu$ is an even integer, we have from (28)

$$
\Omega(\beta,-\alpha)=-i c_{12} \theta_{2}(\beta) \theta_{2}(\alpha)\left[1+\frac{c_{11} \theta_{1}(\alpha)}{c_{12} \theta_{2}(\alpha)}-\frac{c_{22} \theta_{1}(\beta)}{c_{12} \theta_{2}(\beta)}-\frac{\theta_{1}(\alpha) \theta_{1}(\beta)}{\theta_{2}(\alpha) \theta_{2}(\beta)}\right],
$$

and the preceding argument may be applied to show that the bracket on

- Tamarkin, Rendiconti del Circolo Matematico di Palermo, vol.34 (1912). Cf. also Wilder, loc. cit., p. 422. 
the right is asymptotic to 1 for $0<\theta^{\prime} \leqq \theta \leqq \pi-\delta$. An analogous formula is found for $\Omega(\beta,-\alpha)$ when $-\delta \leqq \theta \leqq \theta^{\prime \prime}<0$, while the quantity is uniformly bounded from zero on the semi-circles $\gamma_{m}$. It is clear from this that the subsequent discussion of the case $\nu$ an even integer may be included in the preceding general case by permitting $\eta$ to take the value zero.

Substituting the asymptotic forms in the numerators of formulas (49) we find that when $0 \leqq x \leqq \beta, G(x, t, \rho)$ is bounded uniformly for $\rho$ on the arcs $\gamma_{m}$, and that the asymptotic forms of $G(x, t, \rho)$ may be obtained when $0 \leqq x<\beta$ and $-\alpha<t<\beta$, from the following expressions:

when $-\delta \leqq \theta \leqq \theta^{\prime}<0$,

$$
G(x, t, \rho) \sim\left\{\begin{array}{l}
\frac{i \theta_{2}(x)\left[c_{21} \theta_{1}(t)-c_{11} \theta_{2}(t)\right]}{2 k \rho c_{21}} \text { for } t<x, \\
\frac{i \theta_{2}(t)\left[c_{21} \theta_{1}(x)-c_{11} \theta_{2}(x)\right]}{2 k \rho c_{21}} \text { for } t>x ;
\end{array}\right.
$$

when $0<\theta^{\prime} \leqq \theta \leqq \theta^{\prime \prime}<\eta \pi$,

$$
G(x, t, \rho) \sim\left\{\begin{array}{l}
\frac{i \theta_{1}(x)\left[c_{21} \theta_{1}(t)-c_{11} \theta_{2}(t)\right]}{2 k \rho c_{11}} \text { for } t<x, \\
\frac{i \theta_{1}(t)\left[c_{21} \theta_{1}(x)-c_{11} \theta_{2}(x)\right]}{2 k \rho c_{11}} \text { for } t>x ;
\end{array}\right.
$$

and when $\eta \pi<\theta^{\prime} \leqq \theta \leqq \pi-\delta$,

$$
G(x, t, \rho) \sim\left\{\begin{array}{l}
\frac{i \theta_{1}(x)\left[c_{22} \theta_{1}(t)-c_{12} \theta_{2}(t)\right]}{2 k \rho c_{12}} \text { for } t<x, \\
\frac{i \theta_{1}(t)\left[c_{22} \theta_{1}(x)-c_{12} \theta_{2}(x)\right]}{2 k \rho c_{12}} \text { for } t>x .
\end{array}\right.
$$

8. A lemma. For the discussion of integrals involving $G(x, t, \rho)$ we shall find the following lemma of use. We designate by $\gamma^{(1,2)}$ the portion of the semicircle $\gamma_{m}$ for which $\theta^{\prime} \leqq \theta \leqq \theta^{\prime \prime}$ and set $\rho=r e^{i \theta}$.

LeMma. If $\psi(t)$ and $\Phi(t, \rho)$ are any integrable functions such that for $a \leqq t \leqq b$,

(i) $\psi(t)$ is real and $\psi(t) \geqq \psi>0$;

(ii) $|\Phi(t, \rho)|<M$ for $\theta^{\prime} \leqq \theta \leqq \theta^{\prime \prime}, r>N$, then the integral

$$
I(t)=\int_{\gamma^{(1,2)}} \rho^{l} e^{ \pm i \rho \psi(t)} \Phi(t, \rho) d \rho,
$$

the ambiguous sign being chosen + when $\rho$ is in the upper half plane, and when $\rho$ is in the lower half plane, satisfies the relation $|I(t)| \leqq M_{1} r^{l}$. 
Suppose for the moment that $\gamma^{(1,2)}$ is an arc of the quadrant $0 \leqq \theta \leqq \pi / 2$. Then we have $\sin \theta \geqq \theta / 2$ and hence

and

$$
\left|e^{i \rho \psi(t)}\right|=e^{-r \psi(t) \sin \theta} \leqq e^{-r \psi(t) \theta / 2},
$$

$$
|I(t)| \leqq M r^{l} \int_{\theta^{\prime}}^{\theta^{\prime \prime}} e^{-r \psi(t) \theta / 2} r d \theta<\frac{2 M r^{l}}{\psi} .
$$

If $\gamma^{(1,2)}$ is an arc of the quadrant $\pi / 2 \leqq \theta \leqq \pi$ we have $\sin \theta \geqq(\pi-\theta) / 2$ and the result follows in the same way, as it obviously does also if $-\pi / 2 \leqq \theta \leqq 0$.

9. The function $\phi_{m}(x, t)$ when $x \neq 0$. With the use of the formulas above we shall show first that when $x$ is considered as a parameter, $x>0$,

$$
\left|t^{\nu / 4} \phi_{m}(x, t)\right|<M
$$

for $-\alpha \leqq t \leqq x-\zeta$, or $x+\zeta \leqq t \leqq \beta$, where $\zeta$ is an arbitrary constant subject to $\zeta>0,0 \leqq x-\zeta, x+\zeta \leqq \beta$.

Since $G(x, t, \rho)$ as a function of $x$ or as a function of $t$ satisfies the boundary conditions (16) we see from (48) that

$$
\begin{aligned}
\phi_{m}(-\alpha, t) & \equiv \phi_{m}(\beta, t) \\
& \equiv \phi_{m}(x,-\alpha) \equiv \phi_{m}(x, \beta) \equiv 0 .
\end{aligned}
$$

We may restrict the further discussion therefore to the case $x<\beta,-\alpha<t<\beta$. Furthermore we may choose as the contours $\gamma$ in (48) precisely the semicircles $\gamma_{m}$ of $\S 7$. Consider first the case in which $-\alpha<t \leqq x-\zeta$. Then since by (4) and (9)

$$
t^{\nu / 4}\left[c_{21} \theta_{1}(t)-c_{11} \theta_{2}(t)\right]=e^{i \rho(x-\zeta) k} B_{1}(x, t, \rho) \text { when } \rho \text { is on } \gamma_{m 1},
$$

and

$$
t^{\prime / 4}\left[c_{2, j-1} \theta_{1}(t)-c_{1, j-1} \theta_{2}(t)\right]=e^{-i \rho(x-\zeta) k} B_{j}(x, t, \rho) \text { when } \rho \text { is on } \gamma_{m i}, j=2,3,
$$

we obtain by substituting (51), (51a) and (51b) in (48)

$$
\begin{aligned}
t^{\prime / 4} \phi_{m}(x, t)=\frac{k}{2 \pi} & {\left[\frac{1}{c_{21}} \int_{\gamma_{m 1}} e^{-i \rho[x k-(x-\zeta) k]} B_{1}(x, t, \rho) d \rho\right.} \\
& \left.+\sum_{j=2}^{3} \frac{1}{c_{1, j-1}} \int_{\gamma_{m j}} e^{i \rho[x k-(x-\zeta) k]} B_{j}(x, t, \rho) d \rho\right] .
\end{aligned}
$$

From this it follows by the lemma of $\S 8$ that (53) holds for $-\alpha<t \leqq x-\zeta$. A similar procedure establishes (53) when $x+\zeta \leqq t<\beta$. 
Consider now the integral

$$
I_{m}\left(x, t_{1}, t_{2}\right)=\int_{t_{1}}^{t_{2}} t^{y} \phi_{m}(x, t) d t, \text { for } 0<x<\beta .
$$

We shall show that

$$
\begin{gathered}
\left|I_{m}\left(x, t_{1}, t_{2}\right)\right|<\frac{M}{r_{m}} \quad \text { or } \quad \text { oniformly for }-\alpha \leqq t_{1} \leqq t_{2} \leqq x-\zeta, \\
I_{m}(x,-\alpha, x)=\frac{1}{2}+\epsilon(x, m) ; \\
I_{m}(x, x, \beta)=\frac{1}{2}+\epsilon(x, m) ; \\
\left|I_{m}\left(x, t_{1}, t_{2}\right)\right|<M \text { uniformly for }-\alpha \leqq t_{1} \leqq t_{2}{ }^{-} \leqq \beta,
\end{gathered}
$$

where $\epsilon(x, m)$ is used as a generic symbol for a function which approaches zero as $m \rightarrow \infty$.

The function $G(x, t, \rho)$ as a function of $t$ satisfies the equation (1). Hence, on substituting from (48) in (54) we may write the result

$$
I_{m}\left(x, t_{1}, t_{2}\right)=-\frac{1}{\pi i} \int_{t_{1}}^{t_{2}} \int_{\gamma_{m}} G_{t t}(x, t, \rho) \frac{d \rho}{\rho} d t .
$$

Interchanging the order of integration and integrating we obtain from this

$$
\begin{aligned}
& I_{m}\left(x, t_{1}, t_{2}\right)=-\frac{1}{\pi i} \int_{\gamma_{m}}\left\{G_{t}\left(x, t_{2}, \rho\right)-G_{t}\left(x, t_{1}, \rho\right)\right\} \frac{d \rho}{\rho} \\
& \text { provided }-\alpha \leqq t_{1} \leqq t_{2} \leqq x, \quad \text { or } x \leqq t_{1} \leqq t_{2} \leqq \beta
\end{aligned}
$$

Now from formulas (8) and (13) we find that for $-\alpha \leqq t \leqq x-\zeta$

and

$$
\frac{1}{\rho}\left[c_{21} u_{1}^{\prime}(t)-c_{11} u_{2}^{\prime}(t)\right]=e^{i \rho(x-\zeta)^{k}} B_{1}(x, t, \rho) \text {, for } \rho \text { on } \gamma_{m 1},
$$

$$
\frac{1}{\rho}\left[c_{2, j-1} u_{1}^{\prime}(t)-c_{1, j-1} u_{2}^{\prime}(t)\right]=e^{-i \rho(x-\zeta) k} B_{j}(x, t, \rho), \text { for } \rho \text { on } \gamma_{m i}, j=2,3,
$$

and hence from (51) and the lemma of $\$ 8$

$$
\begin{aligned}
\int_{\gamma_{m}} G_{t}(x, t, \rho) \frac{d \rho}{\rho} & =\int_{\gamma_{m_{1}}} e^{-i \rho[x k-(x-\zeta) k]} B_{1}(x, t, \rho) \frac{d \rho}{\rho} \\
& +\sum_{j=2}^{3} \int_{\gamma_{m} j} e^{i \rho[x k-(x-\zeta) k)]} B_{j}(x, t, \rho) \frac{d \rho}{\rho}=\frac{B(x, t, \rho)}{r_{m}} .
\end{aligned}
$$

Similar considerations lead to the same result when $x+\zeta \leqq t \leqq \beta$. This establishes the relation (55). If we set $\zeta=0$ in (60) the exponential factor 
reduces to unity. Obviously, however, the integrals are still bounded as $m \rightarrow \infty$. It follows that (58) is valid for $t \leqq x$, and a similar discussion establishes it for $t \geqq x$. To deduce (56) we observe that on substituting $t=x$ in the first of formulas (51), (51a), and (51b) and using the asymptotic forms (4) we obtain

$$
\int_{\gamma_{m}} G_{t}(x, x-, \rho) \frac{d \rho}{\rho}=\int_{\gamma_{m}}\left[-\frac{1}{2}+\frac{B(x, \rho)}{\rho}\right] \frac{d \rho}{\rho}=-\frac{\pi i}{2}+\epsilon(x, m) .
$$

Substituting this and the relation (60) for $t=-\alpha$ into (59) we obtain the result (56)

Since the Green's function has the property that

$$
G_{t}(x, x+, \rho)-G_{t}(x, x-, \rho) \equiv 1,
$$

we obtain directly from (61)

$$
\int_{\gamma_{m}} G_{t}(x, x+, \rho) \frac{d \rho}{\rho}=\frac{\pi i}{2}+\epsilon(x, m) .
$$

This together with (60) for $t=\beta$ yields (57).

10. Convergence of the expansion at $x \neq 0$. We prove the following theorem:

THEOREM 5. If $f(x)$ is any function which is integrable in the sense of Lebesgue over the interval $-\alpha \leqq x \leqq \beta$, and if $x$ is any inner point of this interval other than $x=0$, and if further the function $f(t)$ is of bounded variation in any neighborhood of the point $t=x$, then the expansion associated with $f(x)$ converges to

$$
\frac{1}{2}[f(x+)+f(x-)] .
$$

At the end points $x=-\alpha$ or $x=\beta$ the expansion converges to zero.*

From (56) and (57) the expression $\frac{1}{2}[f(x+)+f(x-)]$ may be written in the form

$$
\lim _{m \rightarrow \infty}\left[f(x+) I_{m}(x, x, \beta)+f(x-) I_{m}(x,-\alpha, x)\right] .
$$

On the other hand the sum of the first $m$ terms of the expansion associated with $f(x)$ is by (47)

$$
\int_{-\alpha}^{\beta} t^{\nu} f(t) \phi_{m}(x, t) d t .
$$

It remains to be shown that under the existing hypotheses the difference of these expressions converges to zero. This will follow if

\footnotetext{
* The convergence at $x=0$ is covered by a later theorem.
} 


$$
\lim _{m \rightarrow \infty} D_{j}(x, m)=0 \text {, }
$$$$
i=1,2 \text {, }
$$

where

$$
\begin{aligned}
& D_{1}(x, m)=\int_{-\alpha}^{x} t^{\nu}[f(t)-f(x-)] \phi_{m}(x, t) d t, \\
& D_{2}(x, m)=\int_{x}^{\beta} t^{\nu}[f(t)-f(x+)] \phi_{m}(x, t) d t .
\end{aligned}
$$

Consider the first of these expressions (65). By hypothesis there exists an interval $(x-\zeta, x)$ in which $f(t)$ is of bounded variation. Hence we may write in this interval

$$
f(t)-f(x-)=\chi_{1}(t)-\chi_{2}(t),
$$

where $\chi_{i}(t), j=1,2$, are bounded, positive and decreasing functions and $\chi_{i}(x-)=0$. Hence if $\epsilon$ is arbitrary, $\epsilon>0$, there exists a positive constant $\zeta_{1}<\zeta$ such that

$$
0 \leqq \chi_{j}(t)<\epsilon \text { for } x-\zeta_{1} \leqq t \leqq x, \quad j=1,2 .
$$

We have now

$$
\begin{aligned}
D_{1}(x, m)= & \int_{-\alpha}^{x-\zeta_{1}} t^{\nu}[f(t)-f(x-)] \phi_{m}(x, t) d t \\
& -\sum_{j=1}^{2}(-1)^{j} \int_{x-\zeta_{1}}^{x} t^{\nu} \chi_{j}(t) \phi_{m}(x, t) d t .
\end{aligned}
$$

By virtue of (53), (55) and Lebesgue's* lemma the first of these integrals converges to zero as $m \rightarrow \infty$. By the second law of the mean, however, the remaining integrals in (67) can be written in the form

$$
\chi_{j}\left(x-\zeta_{1}\right) \int_{x-\zeta_{1}}^{t_{j}} t^{v} \phi_{m}(x, t) d t, \text { where } x-\zeta_{1}<t_{j}<x, \quad j=1,2,
$$

and by (58) and (66) this is less than $M \epsilon$. Hence for $m$ sufficiently large

$$
\left|D_{1}(x, m)\right|<3 M \epsilon
$$

and the first of relations (64) is established. The discussion of the expression $D_{2}(x, m)$ is precisely similar and hence we may consider the theorem established.

11. The function $\phi_{m}(x, t)$ when $x=0$. The discussion above was based on the assumption $x \neq 0$. We consider now the excepted case. The function $\phi_{m}(x, t)$ is readily found to be unbounded as a function of $m$ when $x=0$. However, we shall show that when $x=0$ the integral (54) satisfies the relations

* Lebesgue, Annales de la Faculté des Sciences de Toulouse, (3), vol. 1 (1909), p. 52. 


$$
\begin{array}{r}
\left|I_{m}\left(0, t_{1}, t_{2}\right)\right|<\frac{M}{r_{m}^{1 / 2+1 /(2 k)}}, \quad \text { uniformly for }-\alpha \leqq t_{1} \leqq t_{2} \leqq-\zeta \\
\text { or } \quad \zeta \leqq t_{1} \leqq t_{2} \leqq \beta ;
\end{array}
$$

$$
I_{m}(0,-\alpha, 0)=\frac{1}{2}+i \sigma+\epsilon(m), \text { where } \sigma=\frac{1}{2}\left(\eta \tan \frac{\eta^{\prime} \pi}{2 k}-\eta^{\prime} \tan \frac{\eta \pi}{2 k}\right)
$$

$$
\begin{aligned}
& I_{m}(0,0, \beta)=\frac{1}{2}-i \sigma+\epsilon(m) ; \\
& \quad\left|I_{m}\left(0, t_{1}, t_{2}\right)\right|<M \quad \text { uniformly for }-\alpha \leqq t_{1} \leqq t_{2} \leqq \beta .
\end{aligned}
$$

We observe that when $\nu$ is an integer, $\sigma=0$.

The formula (59) is valid for $x=0$. Hence we consider the integral (60) for $x=0$. We have from (51), (51a) and (51b) for $-\alpha \leqq t<0$

$$
\begin{aligned}
\int_{\gamma_{m}} G_{t}(0, t, \rho) \frac{d \rho}{\rho}= & \frac{-i^{\eta}}{2 k}\left[\frac{1}{c_{21}} \int_{\gamma_{m 1}} \frac{u_{2}(0) u_{2}^{\prime}\left(\tau, \rho^{\prime}\right)}{\rho} \frac{d \rho}{\rho}\right. \\
& \left.+\sum_{j=2}^{3} \frac{(-1)^{j}}{c_{1, j-1}} \int_{\gamma_{m j}} \frac{u_{1}(0) u_{-j}^{\prime}(\tau, \rho)}{\rho} \frac{d \rho}{\rho}\right]
\end{aligned}
$$

where $\tau=t e^{-\pi i}$. If $t \leqq-\zeta$ we may substitute the asymptotic forms of $u_{j}^{\prime}\left(\tau, \rho^{\prime}\right)$. This gives to the right hand member the form

$$
\int_{\gamma_{m 1}} e^{-i \rho^{\prime} \tau k} \frac{B_{1}(\tau, \rho)}{\rho^{1 / 2+1 /(2 k)}} d \rho+\int_{\gamma_{m 2}} e^{-i \rho^{\prime} \tau k} \frac{B_{2}(\tau, \rho)}{\rho^{1 / 2+1 /(2 k)}} d \rho+\int_{\gamma_{m}} e^{i \rho^{\prime} \tau k} \frac{B_{3}(\tau, \rho)}{\rho^{1 / 2+1 /(2 k)}} d \rho
$$

to which we may apply the lemma of $\S 8$. From this together with the discussion for $\zeta \leqq t \leqq \beta$, formula (68) follows. On the other hand if $-\zeta \leqq t \leqq 0$, we may turn to formula (12) for the form of $u_{j}^{\prime}\left(\tau, \rho^{\prime}\right)$. The right hand member of (72) is in this case of the form

and relation (71) follows.

$$
\sum_{j=1}^{3} \int_{\gamma_{m j}} B_{j}(\tau, \rho) \frac{d \rho}{\rho}
$$

For the determination of (69) we consider the integral (72) for $t=0-$. Substituting the values given by (15) in (69) and (70) and employing (14) we obtain

$$
\begin{aligned}
& \int_{\gamma_{m}} G_{t}(0,0-, \rho) \frac{d \rho}{\rho}=\frac{-1}{2 \sin \frac{\pi}{2 k}\left\{\left[\frac{c_{11}}{c_{21}}+i e^{-\pi i /(2 k)}\right] \int_{\gamma_{m 1}} \frac{d \rho}{\rho}\right.} \\
& \left.+\left[\frac{c_{21}}{c_{11}}-i e^{\pi i /(2 k)}\right] \int_{\gamma_{m 2}} \frac{d \rho}{\rho}+\left[\frac{c_{22}}{c_{21}}-i e^{\pi i /(2 k)}\right] \int_{\gamma_{m 1}} \frac{d \rho}{\rho}\right\} .
\end{aligned}
$$


But

$$
\int_{\gamma_{m 1}} \frac{d \rho}{\rho}=\delta i, \int_{\gamma_{m},} \frac{d \rho}{\rho}=\eta \pi i, \text { and } \int_{\gamma_{m s}} \frac{d \rho}{\rho}=\left(\eta^{\prime} \pi-\delta\right) i,
$$

while from (10)

$$
\begin{aligned}
& \frac{c_{11}}{c_{21}}=-i\left(\cos \frac{\pi}{2 k}+\tan \frac{\eta \pi}{2 k} \sin \frac{\pi}{2 k}\right), \\
& \frac{c_{21}}{c_{11}}=\quad i\left(\cos \frac{\pi}{2 k}+\tan \frac{\eta^{\prime} \pi}{2 k} \sin \frac{\pi}{2 k}\right), \\
& \frac{c_{22}}{c_{21}}=\quad i\left(\cos \frac{\pi}{2 k}-\tan \frac{\eta \pi}{2 k} \sin \frac{\pi}{2 k}\right) .
\end{aligned}
$$

With these values we obtain

$$
\int_{\gamma_{m}} G_{t}(0,0-, \rho) \frac{d \rho}{\rho}=-\pi i\left\{\frac{1}{2}+\frac{i}{2}\left(\eta \tan \frac{\eta^{\prime} \pi}{2 k}-\eta^{\prime} \tan \frac{\eta \pi}{2 k}\right)\right\},
$$

and this leads directly to (69). The formula (70) follows from (69) because of (62) as in $\$ 9$.

12. The expansion at $x=0$. We prove the following theorem:

THEOREM 6. If $f(x)$ is any function which is of bounded variation on the interval $-\alpha \leqq x \leqq \beta$ then the expansion associated with $f(x)$ converges when $x=0$ to

$$
\left[\frac{1}{2}-i \sigma\right] f(0+)+\left[\frac{1}{2}+i \sigma\right] f(0-),
$$

where $\sigma$ depends on $\nu$ as given in (69), and vanishes when $\nu$ is an integer.

Proceeding as in $\$ 9$, we write (73) in the form (63) with $x=0$, and construct the quantities $D_{i}(0, m)$ given by (65). We must show that (64) follows when $x=0$. Consider $D_{1}(0, m)$. Since $f(t)-f(0-)$ is of bounded variation we may write it in the form

$$
\chi_{1}(t)-\chi_{2}(t),
$$

where $\chi_{j}(t)$ are positive and decreasing, and

$$
\left|\chi_{j}(t)\right|<M
$$

Moreover since $\chi_{i}(0-)=0$, we may choose $\epsilon$ arbitrarily and determine $\zeta$ so that

$$
\left|\chi_{j}(t)\right|<\epsilon, \text { when }-\zeta \leqq t \leqq 0
$$

Then 


$$
D_{1}(0, m)=-\sum_{j=1}^{2}(-1)^{i} \int_{-\alpha}^{-\zeta} t^{v} \chi_{j}(t) \phi_{m}(0, t) d t-\sum_{j=1}^{2}(-1)^{i} \int_{-\zeta}^{0} t^{v} \chi_{j}(t) \phi_{m}(0, t) d t .
$$

Applying the second law of the mean to each of these integrals, we obtain the relation

$$
\begin{aligned}
D_{1}(0, m)=-\sum_{j=1}^{2} & (-1)^{i} \chi_{j}(-\alpha) \int_{-\alpha}^{T_{j}} t^{v} \phi_{m}(0, t) d t \\
& \quad-\sum_{j=1}^{2}(-1)^{i} \chi_{j}(-\zeta) \int_{-\zeta}^{t_{j}} t^{v} \phi_{m}(0, t) d t,
\end{aligned}
$$

where $-\alpha<T_{i}<-\zeta,-\zeta<t_{j}<0$. By (74), (68), (75), and (71), however, this yields

$$
\left|D_{1}(0, m)\right| \leqq \frac{M}{r_{m}^{1 / 2+1 /(2 k)}}+2 M \epsilon<3 M \epsilon \text { for } m \geqq m_{1} .
$$

This establishes the fact that

$$
\lim _{m \rightarrow \infty} D_{j}(0, m)=0,
$$

for $j=1$, and a similar argument establishes it for $j=2$. Hence Theorem 6 is proved.

13. The case $\alpha=0$. We consider now briefly the case in which $x=0$ is an end point of the interval. The boundary conditions are then

$$
u(\beta)=0, \quad u(0)=0,
$$

and the characteristic equation is

$$
\Omega(\beta, 0)=0 .
$$

From (4) and (15) the equation (76) is of the form

$$
\rho^{1 / 2+1 /(2 k)}\left[\theta_{1}(\beta)+i e^{\pi i /(2 k)} \theta_{2}(\beta)\right]=0 .
$$

The observation of $\S 6$ that $\rho=0$ is not a characteristic value is valid for the case in hand, and since the remaining factor in (77) is precisely the first factor of (31) the characteristic values $\rho_{m}$ (or $\lambda_{m}$ ) are given, regardless of the character of $\nu$ by precisely the formula (32) (or (33)) with $j=1$. It follows that the characteristic functions also are given for any value of $\nu$ by the formulas for $u_{1, m}(x)$ in (43), (44), and (45).

Since $x=0$ is now an end point of the interval the expansion converges at this point to zero. For $0<x \leqq \beta$ the discussion is precisely that of $\S \S 6,7,9$ and 10 with $\alpha$ replaced by 0 , and the expansion theorem is accordingly the theorem as stated in $\$ 10$.

UNIVERSITY OF WISCONSIN, Madison, Wis. 